\title{
Intuitionistic Fuzzy Topological BH-Algebras
}

\author{
V. K. Nivedida ${ }^{1}$ and M. Palanivelrajan ${ }^{2}$ \\ ${ }^{1}$ Department of Mathematics, \\ Noble College of Arts and Science for Women, \\ Palavanatham - 626004 \\ ${ }^{2}$ Department of Mathematics, \\ Govt. Arts College, \\ Melur - 625106
}

Received: 11 March 2019

Accepted: 9 April 2019

\begin{abstract}
The intuitionistic fuzzification of a $\mathrm{BH}$-algebra is considered and related results are investigated. The notion of equivalence relations on the family of all intuitionistic fuzzy BHalgebras of a BH-algebra is introduced, and then some properties are discussed. The concept of intuitionistic fuzzy topological $\mathrm{BH}$-algebras is introduced, and some related results are obtained.
\end{abstract} Keywords: intuitionistic fuzzy BH-algebra, intuitionistic fuzzy topological BH-algebra.

2010 Mathematics Subject Classification: 03E72.

\section{Introduction}

Y. Imai and K. Iseki [8] introduced two classes of abstract algebras: BCK-algebras and BCIalgebras. It is known that the class of BCK-algebras is a proper subclass of the class of BCIalgebras. In [6, 7] Q.P. Hu and X. Li introduced a wide class of abstract algebras: BCH-algebras. In 1996, Jun, Roh and Kim introduced the notion of BH-algebra, which is a generalization of BCH-algebras [9]. In 2001, Q. Zhang, E.H. Roh and Y.B. Jun studied the fuzzy theory in BHalgebras [14]. C.H. Park introduced the notion of an interval-valued fuzzy BH-algebra in a BHalgebra and investigate related properties [10]. The concept of a fuzzy set, which was introduced in [13], provides a natural framework for generalizing many of the concepts of general topology to what might be called fuzzy topological spaces. D. H. Foster [5] combined the structure of a 
fuzzy topological spaces with that of a fuzzy group, introduced by A. Rosenfeld [11], to formulate the elements of a theory of fuzzy topological groups. After the introduction of fuzzy sets by L. A. Zadeh [13], several researchers were conducted on the generalizations of the notion of fuzzy sets. The idea of intuitionistic fuzzy set was first published by K. T. Atanassov [1] , as a generalization of the notion of fuzzy sets. In this paper, using the Atanassovs idea, we establish the notion of intuitionistic fuzzy $\mathrm{BH}$-algebras, equivalence relations on the family of all intuitionistic fuzzy $\mathrm{BH}$-algebras, and intuitionistic fuzzy topological $\mathrm{BH}$-algebras which are generalization of the notion of fuzzy topological $\mathrm{BH}$-algebras. We investigate several properties, and show that the $\mathrm{BH}$-homomorphic image and preimage of an intuitionistic fuzzy topological $\mathrm{BH}$-algebra is an intuitionistic fuzzy topological $\mathrm{BH}$-algebra.

\section{Preliminaries}

Definition 2.1 ([9]). Let $\mathrm{X}$ be a set with a binary operation $*$ and a constant 0 . Then $(X, *, 0)$ is called a $\mathrm{BH}$-algebra if it satisfies the following axioms:

1. $x * x=0$,

2. $x * y=0$ and $y * x=0$ imply $x=y$,

3. $x * 0=x$

for all $x, y \in X$.

Definition 2.2. A non-empty set $\mathrm{S}$ of a $\mathrm{BH}$-algebra $\mathrm{X}$ is called a $\mathrm{BH}$-subalgebra of $\mathrm{X}$ if $x * y \in S$ for any $x, y \in X$.

Definition 2.3. A mapping $\theta: X \rightarrow Y$ of BH-algebras is called a BH-homomorphism if $\theta(x *$ $y)=\theta(x) * \theta(y)$ for all $x, y \in X$.

Definition 2.4 ([5]). A fuzzy topology on a set $\mathrm{X}$ is a family $\tau$ of fuzzy sets in $\mathrm{X}$ which satisfies the following conditions:

1. for all $c \in[0,1], k_{c} \in \tau$, where $k_{c}$ has a constant membership function,

2. if $A, B \in \tau$, then $A \cap B \in \tau$,

3. if $A_{j} \in \tau$ for all $j \in J$, then $\bigcup_{j \in J} A_{j} \in \tau$.

The pair $(\mathrm{X}, \tau)$ is called a fuzzy topological space and members of $\tau$ are called open fuzzy sets. 
Definition 2.5. An intuitionistic fuzzy set (IFS for short) D in $\mathrm{X}$ is an object having the form

$$
D=\left\{\left\langle x, \mu_{D}(x), \nu_{D}(x)\right\rangle \mid x \in X\right\}
$$

where the functions $\mu_{D}: X \rightarrow[0,1]$ and $\nu_{D}: X \rightarrow[0,1]$ denote the degree of membership (namely $\mu_{D}(x)$ ) and the degree of nonmembership (namely $\nu_{D}(x)$ ) of each element $x \in X$ to the set $D$, respectively, and $0 \leq \mu_{D}(x)+\nu_{D}(x) \leq 1$ for each $x \in X$.

For the sake of simplicity, we shall use the notation $D=\left\langle x, \mu_{D}, \nu_{D}\right\rangle$ instead of

$$
D=\left\{\left\langle x, \mu_{D}(x), \nu_{D}(x)\right\rangle \mid x \in X\right\} .
$$

Let $f$ be a mapping from a set $X$ to a set $Y$. If

$$
B=\left\{\left\langle y, \mu_{B}(y), \nu_{B}(y)\right\rangle \mid y \in Y\right\}
$$

is an IFS in Y, then the preimage of $B$ under $f$ denoted by $f^{-1}(B)$, is the IFS in $X$ defined by

$$
f^{-1}(B)=\left\{\left\langle x, f^{-1}\left(\mu_{B}\right)(x), f^{-1}\left(\nu_{B}\right)(x)\right\rangle \mid x \in X\right\}
$$

and if

$$
D=\left\{\left\langle x, \mu_{D}(x), \nu_{D}(x)\right\rangle \mid x \in X\right\}
$$

is an IFS in $X$, then the image of $D$ under $f$, denoted by $f(D)$, is the IFS in $Y$ defined by

$$
f(D)=\left\{\left\langle y, f_{\text {sup }} \mu_{D}(y), f_{\text {inf }} \nu_{D}(y)\right\rangle \mid y \in Y\right\},
$$

where

$$
f_{\text {sup }}\left(\mu_{D}\right)(y)= \begin{cases}\sup _{x \in f^{-1}(y)} \mu_{D}(x), & \text { if } f^{-1}(y) \neq \phi \\ 0, & \text { otherwise }\end{cases}
$$

and

$$
f_{\text {inf }}\left(\nu_{D}\right)(y)= \begin{cases}\inf _{x \in f^{-1}(y)} \nu_{D}(x), & \text { if } f^{-1}(y) \neq \phi \\ 1, & \text { otherwise }\end{cases}
$$

for each $y \in Y$.

\section{Intuitionistic fuzzy BH-algebras}

Definition 3.1. Let $X$ be a BH -algebra. An IFS

$$
D=\left\langle x, \mu_{D}, \nu_{D}\right\rangle
$$

in $X$ is called an intuitionistic fuzzy $\mathrm{BH}-$ algebra if it satisfies:

$$
\mu_{D}(x * y) \geq \min \left\{\mu_{D}(x), \mu_{D}(y)\right\}
$$

and

$$
\nu_{D}(x * y) \leq \max \left\{\nu_{D}(x), \nu_{D}(y)\right\} \text { for all } x, y \in X
$$


Example 3.2. Consider a BH-algebra $X=\{0, a, b, c\}$ with the following cayley table:

\begin{tabular}{|l|l|l|l|l|}
\hline$*$ & 0 & $a$ & $b$ & $c$ \\
\hline 0 & 0 & $c$ & 0 & $b$ \\
\hline$a$ & $a$ & 0 & 0 & 0 \\
\hline$b$ & $b$ & $b$ & 0 & $c$ \\
\hline$c$ & $c$ & $c$ & $b$ & 0 \\
\hline
\end{tabular}

Let

$$
D=\left\langle x, \mu_{D}(x), \nu_{D}(x)\right\rangle
$$

be an IFS in $X$ by $\mu_{D}(0)=0.7, \mu_{D}(a)=0.2, \mu_{D}(b)=0.5, \mu_{D}(c)=0.4$ and $\nu_{D}(0)=$ $0.2, \nu_{D}(a)=0.8, \nu_{D}(b)=0.3, \nu_{D}(c)=0.5$. Then

$$
D=\left\langle x, \mu_{D}, \nu_{D}\right\rangle
$$

is an intuitionistic fuzzy $\mathrm{BH}$-algebra.

Proposition 3.3. If an IFS

$$
D=\left\langle x, \mu_{D}, \nu_{D}\right\rangle
$$

in $X$ is an intuitionistic fuzzy BH-algebra of $X$, then

$$
\mu_{D}(0) \geq \mu_{D}(x)
$$

and

$$
\nu_{D}(0) \leq \nu_{D}(x) \text { for all } x \in X \text {. }
$$

Proof. Let $x \in X$. Then

$$
\mu_{D}(0)=\mu_{D}(x * x) \geq \min \left\{\mu_{D}(x), \mu_{D}(x)\right\}=\mu_{D}(x)
$$

and

$$
\nu_{D}(0)=\nu_{D}(x * x) \leq \max \left\{\nu_{D}(x), \nu_{D}(x)\right\}=\nu_{D}(x) .
$$

Theorem 3.4. If $\left\{D_{i} \mid i \in \wedge\right\}$ is an arbitrary family of intuitionistic fuzzy BH-algebras of $X$, then $\cap D_{i}$ is an intuitionistic fuzzy $\mathrm{BH}$-algebra of $X$, where

$$
\cap D_{i}=\left\{\left\langle x, \wedge \mu_{D_{i}}(x), \vee \nu_{D_{i}}(x)\right\rangle \mid x \in X\right\} .
$$

Proof. Let $x, y \in \mathrm{X}$. Then

$$
\wedge \mu_{D_{i}}(x * y) \geq \wedge\left(\min \left\{\mu_{D_{i}}(x), \mu_{D_{i}}(y)\right\}\right)=\min \left\{\wedge \mu_{D_{i}}(x), \wedge \mu_{D_{i}}(y)\right\}
$$

and

$$
\vee \nu_{D_{i}}(x * y) \leq \vee\left(\max \left\{\nu_{D_{i}}(x), \nu_{D_{i}}(y)\right\}\right)=\max \left\{\vee \nu_{D_{i}}(x), \vee \nu_{D_{i}}(y)\right\}
$$

Hence,

$$
\cap D_{i}=\left\langle x, \wedge \mu_{D_{i}}, \vee \nu_{D_{i}}\right\rangle
$$

is an intuitionistic fuzzy BH-algebra of $X$. 
Theorem 3.5. If an IFS $D=\left\langle x, \mu_{D}, \nu_{D}\right\rangle$ in $X$ is an intuitionistic fuzzy BH-algebra of $X$, then so is $\bar{D}$, where $\bar{D}=\left\{\left\langle x, \mu_{D}(x), 1-\mu_{D}(x)\right\rangle \mid x \in X\right\}$.

Proof. It is sufficient to show that $\bar{\mu}_{D}$ satisfies the second condition in Definition 3.1.

Let $x, y \in X$. Then

$$
\begin{aligned}
\mu_{\mathcal{D}}(x * y) & =1-\mu_{D}(x * y) \\
& \leq 1-\min \left\{\mu_{D}(x), \mu_{D}(y)\right\} \\
& =\max \left\{1-\mu_{D}(x), 1-\mu_{D}(y)\right\} \\
& =\max \left\{\mu_{\mathcal{D}}(x), \mu_{\mathcal{D}}(y)\right\}
\end{aligned}
$$

Hence $\mathcal{D}$ is an intuitionistic fuzzy BH-algebra of $X$.

Theorem 3.6. If an IFS $D=\left\langle x, \mu_{D}, \nu_{D}\right\rangle$ in $\mathrm{X}$ is an intuitionistic fuzzy $\mathrm{BH}$-algebra of $X$, then the sets

$$
X_{\mu}:=\left\{x \in X \mid \mu_{D}(x)=\mu_{D}(0)\right\}
$$

and

$$
X_{\nu}:=\left\{x \in X \mid \nu_{D}(x)=\nu_{D}(0)\right\}
$$

are $\mathrm{BH}$-subalgebras of $X$.

Proof. Let $x, y \in X_{\mu}$. Then $\mu_{D}(x)=\mu_{D}(0)=\mu_{D}(y)$, and so $\mu_{D}(x * y)=\min \left\{\mu_{D}(x), \mu_{D}(y)\right\}=$ $\mu_{D}(0)$. By using Proposition 3.3, we know that $\mu_{D}(x * y)=\mu_{D}(0)$ or equivalently $x * y \in X_{\mu}$. Now let $x, y \in X_{\nu}$. Then $\nu_{D}(x * y) \leq \max \left\{\nu_{D}(x), \nu_{D}(y)\right\}=\nu_{D}(0)$, and by applying Proposition 3.3 we conclude that $\nu_{D}(x * y)=\nu_{D}(0)$ and hence $x * y \in X_{\nu}$.

Definition 3.7. Let $D=\left\langle x, \mu_{D}, \nu_{D}\right\rangle$ be an IFS in $X$ and let $t \in[0,1]$. Then the set $U\left(\mu_{D}, t\right):=$ $\left\{x \in X \mid \mu_{D}(x) \geq t\right\}$ (resp. $\left.L\left(\nu_{D}, t\right):=\left\{x \in X \mid \nu_{D}(x) \leq t\right\}\right)$ is called a $\mu$-level t-cut (resp. $\nu$-level t-cut) of D.

Theorem 3.8. If an IFS $D=\left\langle x, \mu_{D}, \nu_{D}\right\rangle$ in $\mathrm{X}$ is an intuitionistic fuzzy BH-algebra of $\mathrm{X}$, then the $\mu$-level t-cut and $\nu$-level t-cut of $\mathrm{D}$ are $\mathrm{BH}$-subalgebras of $\mathrm{X}$ for every $t \in[0,1]$ such that $t \in \operatorname{Im}\left(\mu_{D}\right) \cap \operatorname{Im}\left(\nu_{D}\right)$, which are called a $\mu$-level BH-subalgebra and a $\nu$-level BH-subalgebra respectively.

Proof. Let $x, y \in U\left(\mu_{D}, t\right)$. Then $\mu_{D}(x) \geq t$ and $\mu_{D}(y) \geq t$. It follows that $\mu_{D}(x * y) \geq$ $\min \left\{\mu_{D}(x), \mu_{D}(y)\right\} \geq t$ so that $x * y \in U\left(\mu_{D}, t\right)$. Hence, $U\left(\mu_{D}, t\right)$ is a BH-subalgebra of $X$. Now let $x, y \in L\left(\nu_{D}, t\right)$. Then $\nu_{D}(x * y) \leq \max \left\{\nu_{D}(x), \nu_{D}(y)\right\} \leq t$ and so $x * y \in L\left(\nu_{D}, t\right)$. Therefore, $L\left(\nu_{D}, t\right)$ is a BH-subalgebra of $X$.

Theorem 3.9. Let $D=\left\langle x, \mu_{D}, \nu_{D}\right\rangle$ be an IFS in $X$ such that the sets $U\left(\mu_{D}, t\right)$ and $L\left(\nu_{D}, t\right)$ are BH-subalgebras of $X$. Then $D=\left\langle x, \mu_{D}, \nu_{D}\right\rangle$ is an intuitionistic fuzzy BH-algebra of $X$. 
Proof. Assume that there exist $x_{0}, y_{0} \in X$ such that $\mu_{D}\left(x_{0} * y_{0}\right)<\min \left\{\mu_{D}\left(x_{0}\right), \mu_{D}\left(y_{0}\right)\right\}$.

Let

$$
t_{0}:=1 / 2\left(\mu_{D}\left(x_{0} * y_{0}\right)+\min \left\{\mu_{D}\left(x_{0}\right), \mu_{D}\left(y_{0}\right)\right\}\right) .
$$

Then

$$
\mu_{D}\left(x_{0} * y_{0}\right)<t_{0}<\min \left\{\mu_{D}\left(x_{0}\right), \mu_{D}\left(y_{0}\right)\right\}
$$

and so $x_{0} * y_{0} \notin U\left(\mu_{D}, t_{0}\right)$, but $x_{0}, y_{0} \in U\left(\mu_{D}, t_{0}\right)$. This is a contradiction, and therefore

$$
\mu_{D}(x * y) \geq \min \left\{\mu_{D}(x), \mu_{D}(y)\right\} \text { for all } x, y \in X \text {. }
$$

Now suppose that $\nu_{D}\left(x_{0} * y_{0}\right)>\max \left\{\nu_{D}\left(x_{0}\right), \nu_{D}\left(y_{0}\right)\right\}$ for some $x_{0}, y_{0} \in X$. Taking

$$
S_{0}:=1 / 2\left(\nu_{D}\left(x_{0} * y_{0}\right)+\max \left\{\nu_{D}\left(x_{0}\right), \nu_{D}\left(y_{0}\right)\right\}\right),
$$

then

$$
\max \left\{\nu_{D}\left(x_{0}\right), \nu_{D}\left(y_{0}\right)\right\}<S_{0}<\nu_{D}\left(x_{0} * y_{0}\right) .
$$

It follows that $x_{0}, y_{0} \in L\left(\nu_{D}, S_{0}\right)$ and $x_{0} * y_{0} \notin L\left(\nu_{D}, S_{0}\right)$, a contradiction. Hence

$$
\nu_{D}(x * y) \leq \max \left\{\nu_{D}(x), \nu_{D}(y)\right\} \text { for all } x, y \in X
$$

This completes the proof.

Theorem 3.10. Any BH-subalgebra of $X$ can be realized as both a $\mu$-level BH-subalgebra and a $\nu$-level BH-subalgebra of some intuitionistic fuzzy BH-algebra of $X$.

Proof. Let $\mathrm{S}$ be a BH-subalgebra of $\mathrm{X}$ and let $\mu_{D}$ and $\nu_{D}$ be fuzzy sets in $\mathrm{X}$ defined by

$$
\mu_{D}(x):= \begin{cases}t, & \text { if } x \in S \\ 0, & \text { otherwise }\end{cases}
$$

and

$$
\nu_{D}(x)= \begin{cases}s, & \text { if } x \in S \\ 1, & \text { otherwise }\end{cases}
$$

for all $x \in X$ where $t$ and $s$ are fixed numbers in $(0,1)$ such that $t+s<1$.

Let $x, y \in X$. If $x, y \in S$, then $x * y \in S$. Hence $\mu_{D}(x * y)=\min \left\{\mu_{D}(x), \mu_{D}(y)\right\}$ and $\nu_{D}(x * y)=\max \left\{\nu_{D}(x), \nu_{D}(y)\right\}$. If at least one of $x$ and $y$ does not belong to $S$, then at least one of $\mu_{D}(x)$ and $\mu_{D}(y)$ is equal to 0 , and at least one of $\nu_{D}(x)$ and $\nu_{D}(y)$ is equal to 1 . It follows that

$$
\mu_{D}(x * y) \geq 0=\min \left\{\mu_{D}(x), \mu_{D}(y)\right\}, \nu_{D}(x * y) \leq 1=\max \left\{\nu_{D}(x), \nu_{D}(y)\right\}
$$

Hence $D=\left\langle x, \mu_{D}, \nu_{D}\right\rangle$ is an intuitionistic fuzzy BH-algebra of $X$. Obviously,

$$
U\left(\mu_{D}, t\right)=S=L\left(\nu_{D}, s\right)
$$

This completes the proof. 
Theorem 3.11. Let $\alpha$ be a $\mathrm{BH}$-homomorphism of a $\mathrm{BH}$-algebra $X$ into a $\mathrm{BH}$-algebra $Y$ and $B$ an intuitionistic fuzzy BH-algebra of $Y$. Then $\alpha^{-1}(B)$ is an intuitionistic fuzzy BH-algebra of $X$.

Proof. For any $x, y \in X$, we have

$$
\begin{aligned}
\mu_{\alpha^{-1}}(B)(x * y) & =\mu_{B}(\alpha(x * y)) \\
& =\mu_{B}(\alpha(x) * \alpha(y)) \\
& \geq \min \left\{\mu_{B}(\alpha(x)), \mu_{B}(\alpha(y))\right\} \\
& =\min \left\{\mu_{\alpha^{-1}}(B)(x), \mu_{\alpha^{-1}}(B)(y)\right\}
\end{aligned}
$$

and

$$
\begin{aligned}
\nu_{\alpha^{-1}}(B)(x * y) & =\nu_{B}(\alpha(x * y)) \\
& =\nu_{B}(\alpha(x) * \alpha(y)) \\
& \leq \max \left\{\nu_{B}(\alpha(x)), \nu_{B}(\alpha(y))\right\} \\
& =\max \left\{\nu_{\alpha^{-1}}(B)(x), \nu_{\alpha^{-1}}(B)(y)\right\}
\end{aligned}
$$

Hence $\alpha^{-1}(B)$ is an intuitionistic fuzzy BH-algebra in $X$.

Theorem 3.12. Let $\alpha$ be a BH-homomorphism of a BH -algebra $X$ onto a BH-algebra $Y$. If $D=\left\langle x, \mu_{D}, \nu_{D}\right\rangle$ is an intuitionistic fuzzy BH-algebra of $X$, then $\left.\alpha(D)=\left\langle y, \alpha_{\text {sup }} \mu_{D}\right), \alpha_{\text {inf }}\left(\nu_{D}\right)\right\rangle$ is an intuitionistic fuzzy $\mathrm{BH}$-algebra of $Y$.

Proof. Let $D=\left\langle x, \mu_{D}, \nu_{D}\right\rangle$ be an intuitionistic fuzzy topological BH-algebra in $X$ and let $y_{1}, y_{2} \in$ $Y$. Noticing that

$$
\left\{x_{1} * x_{2} \mid x_{1} \in \alpha^{-1}\left(y_{1}\right) \text { and } x_{2} \in \alpha^{-1}\left(y_{2}\right)\right\} \subseteq\left\{x \in X \mid x \in \alpha^{-1}\left(y_{1} * y_{2}\right)\right\}
$$

we have

$$
\begin{aligned}
\alpha_{\text {sup }}\left(\mu_{D}\right)\left(y_{1} * y_{2}\right) & =\sup \left\{\mu_{D}(x) \mid x \in \alpha^{-1}\left(y_{1} * y_{2}\right)\right\} \\
& \geq \sup \left\{\mu_{D}\left(x_{1} * x_{2}\right) \mid x_{1} \in \alpha^{-1}\left(y_{1}\right) \text { and } x_{2} \in \alpha^{-1}\left(y_{2}\right)\right\} \\
& \geq \sup \left\{\min \left\{\mu_{D}\left(x_{1}\right), \mu_{D}\left(x_{2}\right)\right\} \mid x_{1} \in \alpha^{-1}\left(y_{1}\right) \text { and } x_{2} \in \alpha^{-1}\left(y_{2}\right)\right\} \\
& =\min \left\{\sup \left\{\mu_{D}\left(x_{1}\right) \mid x_{1} \in \alpha^{-1}\left(y_{1}\right)\right\}, \sup \left\{\mu_{D}\left(x_{2}\right) \mid x_{2} \in \alpha^{-1}\left(y_{2}\right)\right\}\right\} \\
& =\min \left\{\alpha_{\text {sup }}\left(\mu_{D}\right)\left(y_{1}\right), \alpha_{\text {sup }}\left(\mu_{D}\right)\left(y_{2}\right)\right\}
\end{aligned}
$$

and

$$
\begin{aligned}
\alpha_{\mathrm{inf}}\left(\nu_{D}\right)\left(y_{1} * y_{2}\right) & =\inf \left\{\nu_{D}(x) \mid x \in \alpha^{-1}\left(y_{1} * y_{2}\right)\right\} \\
& \leq \inf \left\{\nu_{D}\left(x_{1} * x_{2}\right) \mid x_{1} \in \alpha^{-1}\left(y_{1}\right) \text { and } x_{2} \in \alpha^{-1}\left(y_{2}\right)\right\} \\
& \leq \inf \left\{\max \left\{\nu_{D}\left(x_{1}\right), \nu_{D}\left(x_{2}\right)\right\} \mid x_{1} \in \alpha^{-1}\left(y_{1}\right) \text { and } x_{2} \in \alpha^{-1}\left(y_{2}\right)\right\} \\
& =\max \left\{\inf \left\{\nu_{D}\left(x_{1}\right) \mid x_{1} \in \alpha^{-1}\left(y_{1}\right)\right\}, \inf \left\{\nu_{D}\left(x_{2}\right) \mid x_{2} \in \alpha^{-1}\left(y_{2}\right)\right\}\right\} \\
& =\max \left\{\alpha_{\inf }\left(\nu_{D}\right)\left(y_{1}\right), \alpha_{\text {inf }}\left(\nu_{D}\right)\left(y_{2}\right)\right\}
\end{aligned}
$$


Hence $\alpha(D)=\left\langle y, \alpha_{\text {sup }}\left(\mu_{D}\right), \alpha_{\text {inf }}\left(\nu_{D}\right)\right\rangle$ is an intuitionistic fuzzy BH-algebra in $Y$. Let $\Omega(X)$ denote the family of all intuitionistic fuzzy BH-algebras of $X$ and let $t \in[0,1]$. Define binary relations $\tilde{\mu}$ and $\tilde{\nu}$ on $\Omega(X)$ as follows :

$$
A \tilde{\mu} B \Leftrightarrow U\left(\mu_{A}, t\right)=U\left(\mu_{B}, t\right)
$$

and

$$
A \tilde{\nu} B \Leftrightarrow L\left(\nu_{A}, t\right)=L\left(\nu_{B}, t\right),
$$

respectively, for $A=\left\langle x, \mu_{A}, \nu_{A}\right\rangle$ and $B=\left\langle x, \mu_{B}, \nu_{B}\right\rangle \in \Omega(X)$. Then clearly $\tilde{\mu}$ and $\tilde{\nu}$ are equivalence relations on $\Omega(X)$. For any $A=\left\langle x, \mu_{A}, \nu_{A}\right\rangle \in \Omega(X)$, let $[A]_{\mu}$ (resp. $[A]_{\nu}$ )denote the equivalence class of $A=\left\langle x, \mu_{A}, \nu_{A}\right\rangle$ modulo $\tilde{\mu}$ (resp. $\tilde{\nu}$ ), and denote by $\Omega(X) \mid \tilde{\mu}(\operatorname{resp} . \Omega(X) \mid \tilde{\nu})$ the collection of all equivalence classes of A modulo $\tilde{\mu}$ (resp. $\tilde{\nu}) . \Omega(X) \mid \tilde{\mu}:=\left\{[A]_{\mu} \mid A=\left\langle x, \mu_{A}, \nu_{A}\right\rangle \in\right.$ $\Omega(X)\}$ (resp. $\Omega(X) \mid \tilde{\nu}:=\left\{[A]_{\nu} \mid A=\left\langle x, \mu_{A}, \nu_{A}\right\rangle \in \Omega(X)\right\}$. Now, let $\mathrm{S}(\mathrm{X})$ denote the family of all $\mathrm{BH}$-subalgebras of $\mathrm{X}$ and let $t \in[0,1]$. Define maps $\alpha_{t}$ and $\beta_{t}$ from $\Omega(X)$ to $S(X) \cup\{\phi\}$ by $\alpha_{t}(A)=U\left(\mu_{A}, t\right)$ and $\beta_{t}(A)=L\left(\nu_{A}, t\right)$, respectively, for all $\mathrm{A}=\left\langle x, \mu_{A}, \nu_{A}\right\rangle \in \Omega(X)$. Then $\alpha_{t}$ and $\beta_{t}$ are clearly well-defined.

Theorem 3.13. For any $t \in(0,1)$ the maps $\alpha_{t}$ and $\beta_{t}$ are surjective from $\Omega(X)$ to $S(X) \cup\{\phi\}$. Proof. Let $t \in(0,1)$. Note that $0 \tilde{=}\langle x, 0,1\rangle$ is in $\Omega(X)$, where 0 and 1 are fuzzy sets in $X$ defined by $0(\mathrm{x})=0$ and $1(\mathrm{x})=1$ for all $x \in X$. Obviously $\alpha_{t}(0)=\mathrm{U}(0, \mathrm{t})=\phi=\mathrm{L}(1, \mathrm{t})=\beta_{t}(0)$. Let $\mathrm{G}(\neq \phi) \in$ $\mathrm{S}(\mathrm{X})$. For $G^{\sim}=\left\langle x, \chi_{G}, \chi_{G}\right\rangle \in \Omega(X)$, we have $\alpha_{t}(G)=U\left(\chi_{G}, t\right)=\mathrm{G}$ and $\beta_{t}(G)=L\left(\chi_{G}^{\prime}, t\right)=$ G. Hence $\alpha_{t}$ and $\beta_{t}$ are surjective.

Theorem 3.14. The quotient sets $\Omega(X) \mid \tilde{\mu}$ and $\Omega(X) \mid \tilde{\nu}$ are equipotent to $S(X) \cup\{\phi\}$ for every $t \in(0,1)$.

Proof. For $t \in(0,1)$ let $\alpha_{t}^{*}$ (resp. $\beta_{t}^{*}$ ) be a map from $\Omega(X) \mid \tilde{\mu}$ (resp. $\left.\Omega(X) \mid \tilde{\nu}\right)$ to $S(X) \cup\{\phi\}$ defined by $\alpha_{t}^{*}\left([A]_{\mu}\right)=\alpha_{t}[A]$ (resp. $\left.\beta_{t}^{*}\left([A]_{\nu}\right)=\beta_{t}(A)\right)$ for all $A=\left\langle x, \mu_{A}, \nu_{A}\right\rangle \in \Omega(X)$.

If $U\left(\mu_{A}, t\right)=U\left(\mu_{B}, t\right)$ and $L\left(\nu_{A}, t\right)=L\left(\nu_{B}, \mathrm{t}\right)$ for $A=\left\langle x, \mu_{A}, \nu_{A}\right\rangle$ and $B=\left\langle x, \mu_{B}, \nu_{B}\right\rangle \in$ $\Omega(X)$, then $A \tilde{\mu} B$ and $A \tilde{\nu} B$; hence $[A]_{\mu}=[B]_{\mu}$ and $[A]_{\nu}=[B]_{\nu}$. Therefore, the maps $\alpha_{t}^{*}$ and $\beta_{t}^{*}$ are injective. Now let $G(\neq \phi) \in S(X)$. For $G \cong\left\langle x, \chi_{G}, \chi_{G}\right\rangle \in \Omega(X)$, we have $\alpha_{t}^{*}\left([G]_{\mu}\right)=$ $\alpha_{t}\left(G \tilde{)}=U\left(\chi_{G}, t\right)=G\right.$ and $\beta_{t}^{*}\left([G \tilde{]} \nu)=\beta_{t}(G)=L\left(\chi_{G}, t\right)=G\right.$. Finally, for $0 \cong\langle x, 0,1\rangle \in \Omega(X)$ we get

$$
\alpha_{t}^{*}([0] \mu)=\alpha_{t}(0 \tilde{)}=U(0, t)=\phi
$$

and $\beta_{t}^{*}([0] \nu)=\beta_{t}\left(0 \tilde{)}=\mathrm{L}(1, \mathrm{t})=\phi\right.$. This show that $\alpha_{t}^{*}$ and $\beta_{t}^{*}$ are surjective, and we are done. For any $t \in[0,1]$, we define another relation $\mathfrak{R}^{t}$ on $\Omega(X)$ as follows :

$$
(A, B) \in \mathfrak{R}^{t} \Leftrightarrow U\left(\mu_{A}, t\right) \cap L\left(\nu_{A}, t\right)=U\left(\mu_{B}, t\right) \cap L\left(\nu_{B}, t\right) .
$$

For any $A=\left\langle x, \mu_{A}, \nu_{A}\right\rangle, B=\left\langle x, \mu_{B}, \nu_{B}\right\rangle \in \Omega(X)$. Then the relation $\mathfrak{R}^{t}$ is also an equivalence relation on $\Omega(X)$.

Theorem 3.15. For any $t \in(0,1)$, the map $\phi_{t}: \Omega(X) \rightarrow S(X) \cup\{\phi\}$ defined by $\phi_{t}(A)=$ $\alpha_{t}(A) \cap \beta_{t}(A)$ for each $\mathrm{A}=\left\langle x, \mu_{A}, \nu_{A}\right\rangle \in \Omega(X)$ is surjective. 
Proof. Let $t \in(0,1)$. For $0^{\sim}=\langle x, 0,1\rangle \in \Omega(X)$, we get

$$
\phi_{t}\left(0 \tilde{)}=\alpha_{t}\left(0 \tilde{)} \cap \beta_{t}(0)=\cup(0, t) \cap L(1, t)=\phi .\right.\right.
$$

For any $H \in \Omega(X)$, there exists $H \stackrel{\sim}{=}\left\langle x, \chi_{H}, \chi_{H}\right\rangle \in \Omega(X)$ such that

$$
\phi_{t}\left(H \tilde{)}=\alpha_{t}\left(H \tilde { ) } \cap \beta _ { t } \left(H \tilde{)}=\cup\left(\chi_{H}, t\right) \cap L\left(\chi_{H}, t\right)=H .\right.\right.\right.
$$

This completes the proof.

Theorem 3.16. For any $t \in(0,1)$, the quotient set $\Omega(X) \mid \Re^{t}$ is equipotent to $S(X) \cup\{\phi\}$.

Proof. Let $t \in(0,1)$ and let $\phi_{t}^{*}: \Omega(X) \mid \mathfrak{R}^{t} \rightarrow S(X) \cup\{\phi\}$ be a map defined by $\phi_{t}^{*}\left([A] \Re^{t}\right)=\phi_{t}(A)$ for all $[A]_{\mathfrak{R}^{t}} \in \Omega(X) \mid \mathfrak{R}^{t}$. Assume that $\phi_{t}^{*}\left([A]_{\mathfrak{R}^{t}}\right)=\phi_{t}^{*}\left([B]_{\mathfrak{R}^{t}}\right)$ for any $[A]_{\mathfrak{R}^{t}},[B]_{\mathfrak{R}^{t}} \in \Omega(X) \mid \mathfrak{R}^{t}$. Then

$$
\alpha_{t}(A) \cap \beta_{t}(A)=\alpha_{t}(B) \cap \beta_{t}(B) \text {. i.e., } U\left(\mu_{A}, t\right) \cap L\left(\nu_{A}, t\right)=U\left(\mu_{B}, t\right) \cap L\left(\nu_{B}, t\right) \text {. Hence }
$$

$(A, B) \in \mathfrak{R}^{t}$, and so $[A]_{\mathfrak{R}^{t}}=[B]_{\mathfrak{R}^{t}}$. Therefore $\phi_{t}^{*}$ is injective. Now for

$$
0^{\sim}=\langle x, 0,1\rangle \in \Omega(X)
$$

we have

$$
\phi_{t}^{*}\left([0] \mathfrak{R}^{t}\right)=\phi_{t}\left(0 \tilde{)}=\alpha_{t}\left(0 \tilde{)} \cap \beta_{t}(0 \tilde{)}=U(0, t) \cap L(1, t)=\phi .\right.\right.
$$

For

$$
H^{\sim}=\left\langle x, \chi_{H}, \chi_{H}\right\rangle \in \Omega(X)
$$

we get

$$
\phi_{t}^{*}\left([H] \mathfrak{R}^{t}\right)=\phi_{t}\left(H \tilde{)}=\alpha_{t}\left(H \tilde { ) } \cap \beta _ { t } \left(H \tilde{)}=\cup\left(\chi_{H}, t\right) \cap L\left(\chi_{H}, t\right)=H .\right.\right.\right.
$$

Thus $\phi_{t}^{*}$ is surjective. This completes the proof.

\section{Intuitionistic fuzzy topological BH-algebras}

In [4], Coker generalized the concept of fuzzy topological space, first initiated by Chang [3], to the case of intuitionistic fuzzy sets as follows.

Definition 4.1 ([4]). An intuitionistic fuzzy topology (IFT) on a non-empty set X is a family $\Phi$ of IFSs in X satisfying the following axioms:

(T1) $0 \sim 1^{\sim} \in \Phi$,

(T2) $G_{1} \cap G_{2} \in \Phi$ for any $G_{1}, G_{2} \in \Phi$,

(T3) $\cup_{i \in J} G_{i} \in \Phi$ for any family $\left\{G_{i}: i \in J\right\} \subseteq \Phi$.

In this case the pair $(\mathrm{X}, \Phi)$ is called an intuitionistic fuzzy topological space (IFTS for short) and any IFS in $\Phi$ is called an intuitionistic fuzzy open set (IFOS for short) in X.

Definition 4.2. Let $(X, \Phi)$ and $(Y, \Psi)$ be two IFTSs. A mapping $f: X \rightarrow Y$ is said to be intuitionistic fuzzy continuous if the preimage of each IFS in $\Psi$ is an IFS in $\Psi$; and $\mathrm{f}$ is said to be intuitionistic fuzzy open if the image of each IFS in $\Phi$ is an IFS in $\Psi$. 
Definition 4.3. Let $D$ be an IFS in an IFTS $(X, \Psi)$. Then the induced intuitionistic fuzzy topology (IIFT for short) on D is the family of IFSs in $D$ which are the intersection with D of IFOSs in $X$. The IIFT is denoted by $\Psi_{D}$, and the pair $\left(D, \Psi_{D}\right)$ is called an intuitionistic fuzzy subspace of $(X, \Psi)$.

Definition 4.4. Let $\left(D, \Phi_{D}\right)$ and $\left(B, \Psi_{B}\right)$ be intuitionistic fuzzy subspaces of $\operatorname{IFTSs}(X, \Phi)$ and $(Y, \Psi)$, respectively, and let $f: X \rightarrow Y$ be a mapping. Then $f$ is a mapping of $D$ into $B$ if $f(D) \subset B$. Furthermore, $f$ is said to be relatively intuitionistic fuzzy continuous if for each IFS $V_{B} \in \Psi_{B}$, the intersection $f^{-1}\left(V_{B}\right) \cap D$ is an IFS in $\Phi_{D}$; and $f$ is said to be relatively intuitionistic fuzzy open if for each IFS $U_{D} \in \Phi_{D}$, the image $f\left(U_{D}\right)$ is an IFS in $\Psi_{B}$.

Proposition 4.5. Let $\left(D, \Phi_{D}\right)$ and $\left(B, \Psi_{B}\right)$ be intuitionistic fuzzy subspaces of IFTSs $(X, \Phi)$ and $(Y, \Psi)$ respectively, and let $\mathrm{f}$ be an intuitionistic fuzzy continuous mapping of $X$ into $Y$ such that $f(D) \subset B$. Then $f$ is relatively intuitionistic fuzzy continuous mapping of $D$ into $B$.

Proof. Let $V_{B}$ be an IFS in $\Psi_{B}$. Then there exists $V \in \Psi$ such that $V_{B}=V \cap B$. Since $f$ is intuitionistic fuzzy continuous, it follows that $f^{-1}(V)$ is an IFS in $\Phi$. Hence

$$
f^{-1}\left(V_{B}\right) \cap D=f^{-1}(V \cap B) \cap D=f^{-1}(V) \cap f^{-1}(B) \cap D=f^{-1}(V) \cap D
$$

is an IFS in $\Phi_{D}$. This completes the proof.

For any BH-algebra $X$ and any element $a \in X$ we use $a_{r}$ to denote the selfmap of $X$ defined by $a_{r}(x)=x * a$ for all $x \in X$.

Definition 4.6. Let $X$ be BH-algebra, $\Phi$ an IFT on $X$ and $D$ an intuitionistic fuzzy BH- algebra with IIFT $\Phi_{D}$. Then $D$ is called an intuitionistic fuzzy topological BH-algebra if for each $a \in \mathrm{X}$ the mapping

$$
a_{r}:\left(D, \Phi_{D}\right) \rightarrow\left(D, \Phi_{D}\right), x \mapsto x * a,
$$

is relatively intuitionistic fuzzy continuous.

Theorem 4.7. Given BH-algebras $X$ and $Y$, and a BH-homomorphism $\alpha: X \rightarrow Y$, let $\Phi$ and $\Psi$ be the IFTs on $X$ and $Y$, respectively such that $\Phi=\alpha^{-1}(\Psi)$. If $B$ is an intuitionistic fuzzy topological BH-algebra in $\mathrm{Y}$, then $\alpha^{-1}(B)$ is an intuitionistic fuzzy topological $\mathrm{BH}$-algebra in $\mathrm{X}$.

Proof. Let $a \in X$ and let $U$ be an IFS in $\Phi_{\alpha^{-1}}(B)$. Since $\alpha$ is an intuitionistic fuzzy continuous mapping of $(X, \Phi)$ into $(Y, \Psi)$, it follows from Proposition 4.5 that $\alpha$ is a relatively intuitionistic fuzzy continuous mapping of $\left(\alpha^{-1}(B), \Phi_{\alpha^{-1}}(B)\right)$ into $\left(B, \Psi_{B}\right)$. Note that there exists an IFS $V$ in $\Psi_{B}$ such that $\alpha^{-1}(V)=U$. Then

$$
\begin{aligned}
\mu_{a_{r}^{-1}}(U)(x) & =\mu_{U}\left(a_{r}(x)\right)=\mu_{U}(x * a)=\mu_{\alpha^{-1}(V)}(x * a) \\
& =\mu_{V}(\alpha(x * a))=\mu_{V}(\alpha(x) * \alpha(a))
\end{aligned}
$$

and

$$
\begin{aligned}
\nu_{a_{r}^{-1}}(U)(x) & =\nu_{U}\left(a_{r}(x)\right)=\nu_{U}(x * a)=\nu_{\alpha^{-1}(V)}(x * a) \\
& =\nu_{V}(\alpha(x * a))=\nu_{V}(\alpha(x) * \alpha(a))
\end{aligned}
$$


Since $B$ is an intuitionistic fuzzy topological $\mathrm{BH}$-algebra in $Y$, the mapping

$$
b_{r}:\left(B, \Psi_{B}\right) \rightarrow\left(B, \Psi_{B}\right), y \mapsto y * b
$$

is relatively intuitionistic fuzzy continuous for every $b \in Y$. Hence,

$$
\begin{aligned}
\mu_{a_{r}^{-1}}(U)(x) & =\mu_{V}(\alpha(x) * \alpha(a))=\mu_{V}(\alpha(a) r(\alpha(x))) \\
& =\mu_{\alpha(a) r(V)^{-1}}(\alpha(x))=\mu_{\alpha\left(\alpha(a) r^{-1}(V)\right)}^{-1}(x)
\end{aligned}
$$

and

$$
\begin{aligned}
\nu_{a_{r}^{-1}}(U)(x) & =\nu_{V}(\alpha(x) * \alpha(a))=\nu_{V}(\alpha(a) r(\alpha(x))) \\
& =\nu_{\alpha(a) r(V)^{-1}}(\alpha(x))=\nu_{\alpha\left(\alpha(a) r^{-1}(V)\right)}^{-1}(x)
\end{aligned}
$$

Therefore

$$
a_{r}^{-1}(U)=\alpha^{-1}\left(\alpha(a) r^{-1}(V)\right)
$$

and so

$$
a_{r}^{-1}(U) \cap \alpha^{-1}(B)=\alpha^{-1}\left(\alpha(a) r^{-1}(V)\right) \cap \alpha^{-1}(B)
$$

is an IFS in $\Phi_{\alpha^{-1}}(B)$.

This completes the proof.

Theorem 4.8. Given BH-algebras $X$ and $Y$, and a BH-isomorphism $\alpha$ of $X$ to $Y$, let $\Phi$ and $\Psi$ be the IFTs on $\mathrm{X}$ and $\mathrm{Y}$ respectively such that $\alpha(\Phi)=\Psi$. If $D$ is an intuitionistic fuzzy topological $\mathrm{BH}$-algebra in $X$, then $\alpha(D)$ is an intuitionistic fuzzy topological $\mathrm{BH}$-algebra in $Y$.

Proof. It is sufficient to show that the mapping

$$
b_{r}:\left(\alpha(D), \Psi_{\alpha(D)}\right) \rightarrow\left(\alpha(D), \Psi_{\alpha(D)}\right), y \mapsto y * b
$$

is relatively intuitionistic fuzzy continuous for each $b \in Y$. Let $U_{D}$ be an IFS in $\Psi_{D}$. Then there exists an IFS $U$ in $\Phi$ such that $U_{D}=U \cap D$. Since $\alpha$ is one-one, it follows that

$$
\alpha\left(U_{D}\right)=\alpha(U \cap D)=\alpha(U) \cap \alpha(D)
$$

which is an IFS in $\Psi_{\alpha(D)}$. This shows that $\alpha$ is relatively intuitionistic fuzzy open.

Let $V_{\alpha}(D)$ be an IFS in $\Psi_{\alpha}(D)$. The surjectivity of $\alpha$ implies that for each $b \in Y$ there exists $a \in X$ such that $b=\alpha(a)$. Hence

$$
\begin{aligned}
\mu^{-1} \alpha(b r(V \alpha(D))(x) & =\mu_{\alpha^{-1}\left(\alpha(a) r^{-1}(V \alpha(D))\right.}(x) \\
& =\mu_{\alpha(a) r^{-1}(V \alpha(D))}(\alpha(x)) \\
& =\mu_{V \alpha(D)}(\alpha(a) r(\alpha(x))) \\
& =\mu_{V \alpha(D)}(\alpha(x) * \alpha(a)) \\
& =\mu_{V \alpha(D)}(\alpha(x * a)) \\
& =\mu_{\alpha^{-1}(V \alpha(D))}(x * a) \\
& =\mu_{\alpha^{-1}(V \alpha(D))}\left(a_{r}(x)\right) \\
& =\mu_{a_{r}^{-1}\left(\alpha^{-1}(V \alpha(D))\right.}(x)
\end{aligned}
$$




$$
\begin{aligned}
\nu^{-1} \alpha(b r(V \alpha(D))(x) & =\nu_{\alpha^{-1}\left(\alpha(a) r^{-1}(V \alpha(D))\right.}(x) \\
& =\nu_{\alpha(a) r^{-1}(V \alpha(D))}(\alpha(x)) \\
& =\nu_{V \alpha(D)}(\alpha(a) r(\alpha(x))) \\
& =\nu_{V \alpha(D)}(\alpha(x) * \alpha(a)) \\
& =\nu_{V \alpha(D)}(\alpha(x * a)) \\
& =\nu_{\alpha^{-1}(V \alpha(D))}(x * a) \\
& =\nu_{\alpha^{-1}(V \alpha(D))}\left(a_{r}(x)\right) \\
& =\nu_{a_{r}^{-1}\left(\alpha^{-1}(V \alpha(D))\right.}(x)
\end{aligned}
$$

Therefore

$$
\alpha^{-1}\left(b_{r}^{-1}\left(V_{\alpha(D)}\right)=a_{r}^{-1}\left(\alpha^{-1}\left(V_{\alpha(D)}\right)\right) .\right.
$$

By hypothesis, the mapping

$$
a_{r}:\left(D, \Phi_{D}\right) \rightarrow\left(D, \Phi_{D}\right), x \mapsto x * a
$$

is relatively intuitionistic fuzzy continuous and $\alpha$ is a relatively intuitionistic fuzzy continuous map:

$$
\left(D, \Phi_{D}\right) \rightarrow\left(\alpha(D), \Psi_{\alpha(D)}\right)
$$

Thus

$$
\alpha^{-1}\left(b_{r}^{-1}\left(V_{\alpha(D)}\right)\right) \cap D=a_{r}^{-1}\left(\alpha^{-1}\left(V_{\alpha(D)}\right)\right) \cap D
$$

is an IFS in $\Phi_{D}$. Since $\alpha$ is relatively intuitionistic fuzzy open,

$$
\alpha\left(\alpha^{-1}\left(b_{r}^{-1}\left(V_{\alpha(D)}\right)\right) \cap D\right)=b_{r}^{-1}\left(V_{\alpha(D)}\right) \cap \alpha(D) .
$$

is an IFS in $\Psi_{\alpha(D)}$. This completes the proof.

\section{References}

[1] Atanassov, K. (1986). Intuitionistic fuzzy sets. Fuzzy Sets and Systems, 35 , 87-96.

[2] Atanassov, K.T. (2015) One new algebraic object, Int.J.Bioautomation, 20 (S1), S75-S81.

[3] Chang, C. (1968). Fuzzy topological spaces. J. Math. Anal. Appl., 24, 182-190.

[4] Coker, D. (1997). An introduction to intuitionistic fuzzy topological spaces. Fuzzy Sets and Systems, 88, 81-89.

[5] Foster, D. H. (1979). Fuzzy topological groups. J. Math. Anal. Appl., 67, 549-564

[6] Hu, Q. P. \& Li, X. (1983). On BCH-algebras. Math. Seminar Notes, 11, 313-320.

[7] Hu, Q. P. \& Li, X. (1985). On Proper BCH-algebras. Math. Japan., 30, 659-661. 
[8] Imai, Y \& Iseki, K. (1966). On axiom systems of propositional calculi XIV. Proc. Japan Academy, 42, 19-22

[9] Jun, Y. B., Roh, E. H. \& Kim, H. S. (1998) On BH-algebras, Sci. Mathematicae 1, 347-354.

[10] Park, C. H. (2006). Interval-valued fuzzy ideal in BH-algebras. Advance in Fuzzy Set and System, 1, 231-240.

[11] Rosenfeld, A. (1971). Fuzzy groups. J. Math. Anal. Appl., 35, 512-517.

[12] Senapati, T., Bhowmik, M., \& Pal, M. (2013) Intuitionistic fuzzy translations of intuitionistic fuzzy H-ideals in BCK/BCI algebras, Notes on Intuitionistic Fuzzy sets, 19(1) , 32-47.

[13] Zadeh, L. A. (1965). Fuzzy sets. Information and Control, 8(3), 338-353.

[14] Zhang, Q., Roh, E. H., \& Jun, Y. B. (2001). On Fuzzy BH-algebras. J. Huanggang, Normal Univ., 21, 14-19. 